\title{
Tumores del estroma gastrointestinal (GIST): serie del Hospital Central de la Defensa Gómez Ulla
}

\author{
Bodega Quiroga I. ${ }^{1 a}$, Tejedor Togores P. ${ }^{2 a}$, Sáez García MA. ${ }^{\text {b }}$, Peraza Casajús JM. ${ }^{\text {a }}$, \\ Gutiérrez Ortega C. ${ }^{\text {cc }}$, Rosado Dawid N. ${ }^{2 d}$, Serrano Muñoz A. ${ }^{4 a}$
}

Sanid. mil. 2013; 69 (3): 173-181; ISSN: 1887-8571

\begin{abstract}
RESUMEN
Objetivo: Los tumores del estroma gastrointestinal (GIST) se han diferenciado hace poco más de una década de los tumores de músculo liso y de origen neural gracias a métodos de identificación inmunohistoquímica (CD117). Al mismo tiempo, la introducción del Mesylato de Imatinib, fármaco empleado en el tratamiento de la leucemia mieloide crónica (LMC), ha mejorado la expectativa de vida, no sólo en GIST irresecables o metastáticos, sino también para aquéllos de intermedio o alto grado de malignidad como terapia adyuvante e incluso se plantea como tratamiento neoadyuvante. El objetivo de esta comunicación es estudiar los GIST diagnosticados e intervenidos quirúrgicamente en el Servicio de Cirugía General y del Aparato Digestivo del Hospital Central de la Defensa «Gómez Ulla» (Madrid) en un periodo de 9 años y realizar una revisión de la literatura enfocada fundamentalmente a los avances en el tratamiento. Material y métodos: Estudio retrospectivo observacional de los pacientes diagnosticados de GIST e intervenidos quirúrgicamente en nuestro Servicio de 2003 a 2012. Se estudia el motivo de consulta inicial, la localización, el grado histológico y el tipo de intervención quirúrgica realizado. Resultados: Se encontraron 11 pacientes entre Noviembre de 2003 y Abril de 2012 , todos hombres. La edad media fue de 65'17 años (rango, 53-84). Hay que destacar que en 8 casos (72'7 \%) el hallazgo fue incidental, sin ninguna sintomatología previa. La localización más frecuente fue el estómago en 6 casos (54'5\%), y en el intestino delgado en $5\left(45^{\prime} 5 \%\right)$. En cuanto al riesgo de malignidad, 5 casos (45, $\left.5 \%\right)$ fueron de bajo grado, $4\left(36^{\prime} 4 \%\right)$ de grado intermedio, $1(9 \%)$ de muy bajo grado y $1\left(9^{\prime} 1 \%\right)$ de alto grado de malignidad. La técnica quirúrgica empleada fue la gastrectomía parcial en 6 casos (54’5\%) seguida de la resección intestinal segmentaria en 5 casos (45 5\%). Conclusiones: La incidencia anual de tumores GIST intervenidos en nuestro servicio es de 1 nuevo caso/año. Las localizaciones han sido el estómago y el intestino delgado. Todos los tumores de grado bajo se han beneficiado de cirugía, con tasas de supervivencia excelentes sin tratamiento adicional. Hasta conocer los resultados de los estudios en curso sobre la terapia neoadyuvante con Imatinib, parece que en tumores GIST operables se debería realizar primero cirugía y valorar, según los hallazgos intraoperatorios, la adyuvancia y, en casos de tumores solo parcialmente resecables o metastásicos, la neoadyuvancia.
\end{abstract}

PALABRAS CLAVE: Tumores del estroma gastrointestinal, Imatinib, Receptor c-kit, Neoadyuvancia.

\section{Gastrointestinal stromal tumors (GIST): series of the Defense Central Hospital «Gómez Ulla»}

SUMMARY: Introduction: Since a little more than a decade the gastrointestinal stromal tumors (GIST) have been differentiated from the tumors of smooth muscle and of neural origin by immunohistochemical methods (CD117). Simultaneously the introduction of imatinib mesylate, utilized in the treatment of chronic myeloid leukemia (CML), has improved the life expectancy, not only in unresectable or metastatic GIST, but also in those of high or intermediate degree of malignancy as adjuvant therapy or even as neoadjuvant therapy. Objective: To study the GIST diagnosed and operated in the Department of General and Digestive Surgery of the Defense Central Hospital in 9 years and to review the literature mainly focused on therapeutic advances. Material and Methods: Retrospective observational study of the GIST patients diagnosed and operated in our Department from 2003 to 2012 . The reason for the initial consultation, localization, histologic grade and type of surgery performed are analyzed. Results: Eleven patients, all male, were diagnosed between November 2003 and April 2012. Average age was 65.17 (range 53-84). It must be emphasized that in 8 cases $(72.7 \%)$ the finding was incidental, without any previous symptoms. The most frequent localization was the stomach in 6 cases $(54.5 \%)$ and the small intestine in $5(45.5 \%)$. As far as the malignancy risk is concerned 5 cases $(45.5 \%)$ were low grade, 4 cases $(36.4 \%)$ intermediate grade, 1 case $(9 \%)$ very low grade and 1 case $(9.1 \%)$ high malignancy grade. The surgical technique utilized was partial gastrectomy in 6 cases $(54.5 \%)$ followed by segmental intestinal resection in 5 cases (45.5\%). Conclusions: The annual inciden-

${ }^{1}$ Cte. Médico.

${ }^{2}$ Médico civil.

${ }^{3}$ Biólogo civil.

${ }^{4}$ Col. Médico.

a Servicio de Cirugía General y Aparato Digestivo.

b Servicio de Anatomía Patológica.

c Servicio de Medicina Preventiva.

d Servicio de Aparato Digestivo.

Hospital Central de la Defensa «Gómez Ulla». Madrid.

Dirección para correspondencia: ibodqui@oc.mde.es

Recibido: 7 de febrero de 2013

Aceptado: 16 de abril de 2013 ce of GIST tumors operated in our Department is 1 new case / year. The localizations were in the stomach and small intestine. All low grade tumors have benefited from surgery with excellent survival rates without additional treatment. Until the results of the ongoing studies about neoadjuvant therapy with imatinib are known, it seems that operable GIST tumors should first undergo surgery and assess, in accordance with intraoperative findings, the adjuvancy and for only partially resectable o metastatic tumors, the neoadjuvancy.

KEYWORDS: Gastrointestinal stromal tumors; Imatinib; c-kit receptor; Neoadjuvant therapy. 


\section{INTRODUCCIÓN}

Hasta hace poco más de una década (aunque se conocen desde hace más de 20), los tumores del estroma gastrointestinal (GIST) constituían un grupo heterogéneo de neoplasias que incluían los leiomiomas, leiomiosarcomas, leiomioblastomas y tumores nerviosos autonómicos (Schwanomas). Representan aproximadamente el 0,5-3\% de todos los tumores primarios del tracto gastrointestinal, tan sólo el 5\% de los sarcomas viscerales, pero el $80 \%$ de los tumores malignos que se originan en el tracto mesenquimal ${ }^{1}$.

Se trataba de entidades muy resistentes a quimioterapia y radioterapia convencionales. Hoy en día, gracias a métodos de identificación inmunohistoquímica (c-kit) se ha observado que los GIST expresan CD117 y, en ocasiones, CD34. Estos hallazgos llevaron a pensar que determinadas mutaciones del oncogén c-kit, producen la activación y proliferación incontrolada del receptor de tirosin-quinasa en los GIST. Se piensa que derivan de las células intersticiales de Cajal, presentes en los plexos mientéricos. Gracias al descubrimiento de nuevos fármacos que actúan sobre el receptor CD117, se han convertido en el primer modelo de tumor sólido que puede tratarse de forma eficaz con Imatinib. Por tanto, no sólo es importante el receptor KIT para el diagnóstico, sino que es precisamente sobre el receptor donde actúa el fármaco.

\section{OBJETIVO}

El objetivo de este trabajo es presentar la experiencia que en este tipo de tumores hemos tenido en el Hospital Central de la Defensa Gómez Ulla y, tras una revisión de la literatura, examinar el papel actual de la cirugía e Imatinib en el manejo de los GIST.

\section{MATERIAL Y MÉTODOS}

Estudio retrospectivo observacional en el que se revisaron las historias clínicas de los 11 pacientes diagnosticados e interveni-

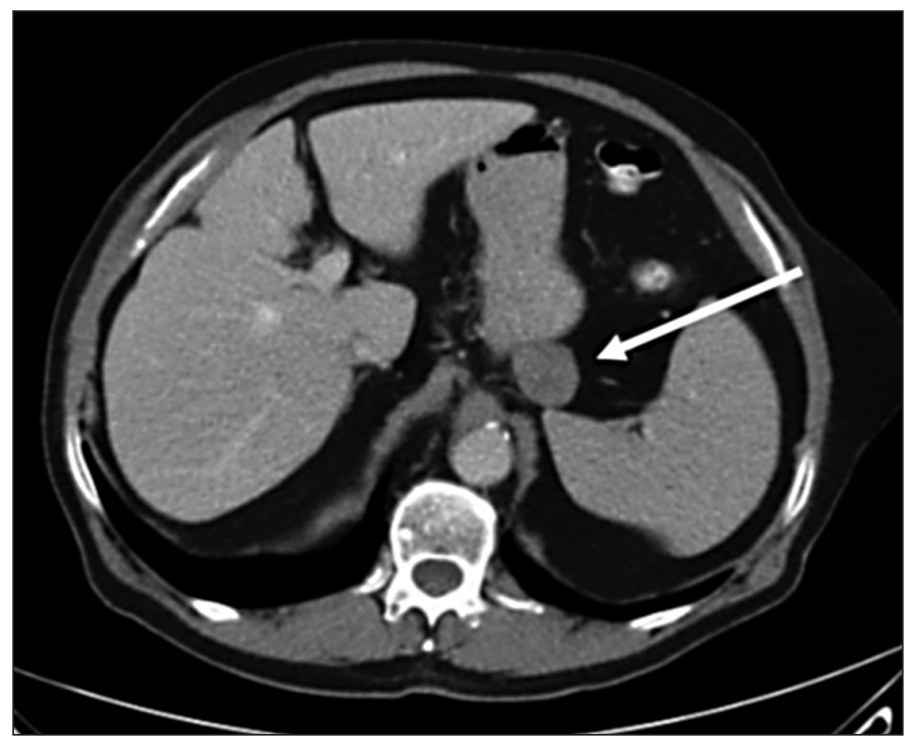

Figura 1. TC abdominal de GIST gástrico.

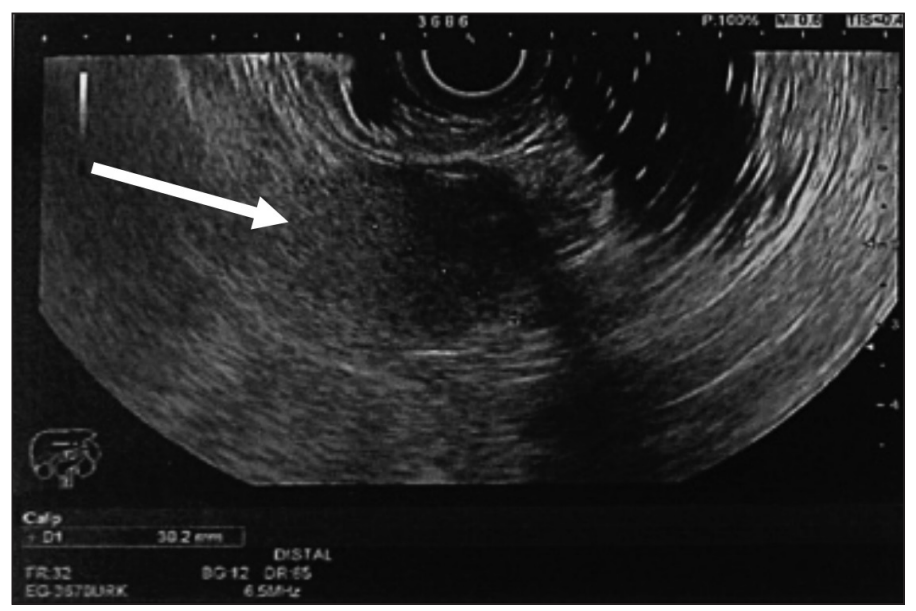

Figura 2. Ecoendoscopia de GIST gástrico.

dos quirúrgicamente de GIST en el Servicio de Cirugía General y del Aparato Digestivo del Hospital Central de la Defensa Gómez Ulla desde el mes de Noviembre de 2003 hasta el mes de Abril de 2012.

Tabla 1. Resultados de la serie.

\begin{tabular}{|c|c|c|c|c|c|}
\hline Caso & Edad & Sexo & Localización & Manifestación & Malignidad \\
\hline 1 & 66 & Hombre & ID & Dolor abdominal & Intermedio \\
\hline 2 & 78 & Hombre & ID & Incidental & Muy bajo \\
\hline 3 & 53 & Hombre & ID & Anemia (SOH) & Alto \\
\hline 4 & 80 & Hombre & Estómago & Incidental & Bajo \\
\hline 5 & 61 & Hombre & ID & Incidental & Intermedio \\
\hline 6 & 84 & Hombre & Estómago & HDA & Intermedio \\
\hline 7 & 79 & Hombre & Estómago & Incidental & Bajo \\
\hline 8 & 76 & Hombre & Estómago & Incidental & Bajo \\
\hline 9 & 65 & Hombre & Estómago & Incidental & Bajo \\
\hline 10 & 77 & Hombre & Estómago & Incidental & Bajo \\
\hline 11 & 63 & Hombre & ID & Incidental & Intermedio \\
\hline
\end{tabular}

* ID: Intestino delgado; SOH: Sangre oculta en heces; HDA: Hemorragia digestiva alta. 
Los criterios de inclusión para el estudio fueron: diagnóstico anatomopatológico de GIST y tinción inmunohistoquímica positiva para CD117 y/o CD117 negativo con alta sospecha de GIST y DOG1 positivo.

Como métodos de diagnóstico se empleó Tomografía Computarizada (TC) abdominal en todos los casos (Fig. 1). Se utilizaron también la endoscopia, ecografía, ecoendoscopia (Fig. 2), y Resonancia Magnética Nuclear dependiendo de la sospecha clínica y la localización del tumor.

Se revisó la base de datos del Servicio de Anatomía Patológica y, con objeto de unificar los criterios y homogeneizar el grupo, se añadieron o repitieron las técnicas de inmunohistoquímica de los marcadores CD117 (c-kit), DOG1, CD34, Actina de músculo liso, Desmina, Vimentina y Proteína S-100. Como marcador del índice de proliferación se realizó inmunotinción con Ki67. Se contabilizó el número de mitosis por 50 campos de gran aumento (CGA). Los datos se muestran en la tabla 1, incluyendo el tamaño del tumor, la localización y el grado.

\section{RESULTADOS}

Se encontraron 11 pacientes entre Noviembre de 2003 y Abril de 2012, todos ellos hombres. La edad media fue de $65^{\prime} 17$ años (rango: 53-84). Hay que destacar que en 8 casos (72,7 \%) el hallazgo fue incidental, sin ninguna sintomatología previa. En los otros tres pacientes el modo de presentación fue hemorragia digestiva alta, dolor abdominal y anemia.

La localización más frecuente fue el estómago en 6 casos $\left(54^{\prime} 5 \%\right)$, y en el intestino delgado en $5\left(45^{\prime} 5 \%\right)$ (Tabla 1$)$.

Todos los tumores fueron DOG1 positivos. El 90’9\% (10) fueron CD117 positivos. El CD34 y la actina fueron positivos en el $363 \%$ (4) y 54 '5\% (6) respectivamente. Todos se tiñeron con vimentina y ninguno lo hizo con desmina ni S100 (Tabla 2; Figura 3).

En cuanto al riesgo de malignidad, según los criterios de consenso de pronóstico de Fletcher ${ }^{2}$ (Tabla 3 ), clasificación que combina tamaño y número de mitosis, 5 casos $(45,5 \%)$ fueron de bajo grado, $4\left(36{ }^{\circ} \%\right)$ de grado intermedio, 1 (9\%) de muy bajo grado y $1\left(9^{\prime} 1 \%\right)$ de alto grado de malignidad.
La técnica quirúrgica empleada fue la gastrectomía parcial en 6 casos $(54,5 \%)$ seguida de la resección intestinal segmentaria en 5 casos $\left(45^{\prime} 5 \%\right)$. Uno de los pacientes que presentaba un GIST de localización gástrica fue operado por laparoscopia en el año 2012; el resto de pacientes fueron sometidos a cirugía abierta.

En el seguimiento, todos los pacientes con tumores de bajo y muy bajo grado están en la actualidad asintomáticos y libres de enfermedad. Hay un paciente con GIST de riesgo intermedio con origen en el intestino delgado que es el único que ha seguido tratamiento con Imatinib a dosis de $400 \mathrm{mg} /$ día. Han fallecido tres pacientes: el que presentó un tumor de alto grado en localización intestinal y dos de grado intermedio con localización gástrica e intestinal respectivamente. Los pacientes fallecidos presentaban, según la clasificación de Fletcher, tumores de intermedio o alto riesgo de malignidad.

\section{DISCUSIÓN}

La incidencia de los tumores GIST no está del todo bien definida. Se estima que, en los EEUU, se diagnostican anualmente entre 5.000-6.000 casos, lo que supone 10-20 casos nuevos por millón de habitantes ${ }^{2,3}$. La incidencia anual de tumores GIST intervenidos en nuestro servicio es difícil de calcular, puesto que la población de referencia ha cambiado en los últimos años de 30.000-50.000 a los 120.000-130.000 individuos actuales. Esto supondría una incidencia aproximada de 1 caso por 100.000 habitantes-año aunque hemos observado una tendencia al alza en los últimos 3 años, probablemente debido al cambio de población a la que se da asistencia al haber pasado a dar cobertura a un área sanitaria de la Comunidad de Madrid. Los GIST son más frecuentes en hombres que en mujeres y la edad media de presentación son los 60 años, lo que concuerda con nuestros resultados. De hecho todos los pacientes de nuestra serie son varones y la edad media es de 65'17 años. Se han descrito en todos los grupos de edad pero son muy infrecuentes por debajo de los 30 años. La localización más frecuente, según la mayoría de las series, es el estómago, seguido del intestino delgado (Fig. 4), que

Tabla 2. Resultados del estudio anatomopatológico.

\begin{tabular}{|c|c|c|c|c|c|c|c|c|c|c|c|}
\hline Caso & Loc. & $\begin{array}{c}\text { Tamaño } \\
\text { (cm.) }\end{array}$ & $\begin{array}{c}\text { Mitosis } \\
(* 50 \text { CGA })\end{array}$ & CD 117 & DOG1 & CD 34 & Actina & Desmina & Vimentina & $\mathrm{S} 100$ & Grado \\
\hline 1 & ID & 8,5 & 1 & + & + & + & + & - & + & - & Intermedio \\
\hline 2 & ID & 1,5 & 0 & + & + & - & + & - & + & - & Muy bajo \\
\hline 3 & ID & 9 & $>10$ & + & + & - & - & - & + & - & Alto \\
\hline 4 & Estómago & 4,5 & 2 & + & + & - & - & - & + & - & Bajo \\
\hline 5 & ID & 5 & 5 & + & + & - & - & - & + & - & Intermedio \\
\hline 6 & Estómago & 4 & 3 & + & + & + & + & - & + & - & Intermedio \\
\hline 7 & Estómago & 4,5 & 2 & + & + & - & - & - & + & - & Bajo \\
\hline 8 & Estómago & $2 \times 1,5$ & 1 & - & + & - & - & - & + & - & Bajo \\
\hline 9 & Estómago & 2,3 & 1 & + & + & + & + & - & + & - & Bajo \\
\hline 10 & Estómago & $4 \times 2,5$ & 3 & + & + & + & + & - & + & - & Bajo \\
\hline 11 & ID & 9 & 2 & + & + & - & + & - & + & - & Intermedio \\
\hline
\end{tabular}

*Loc: localización. (*50CGA): mitosis por 50 campos de gran aumento. 


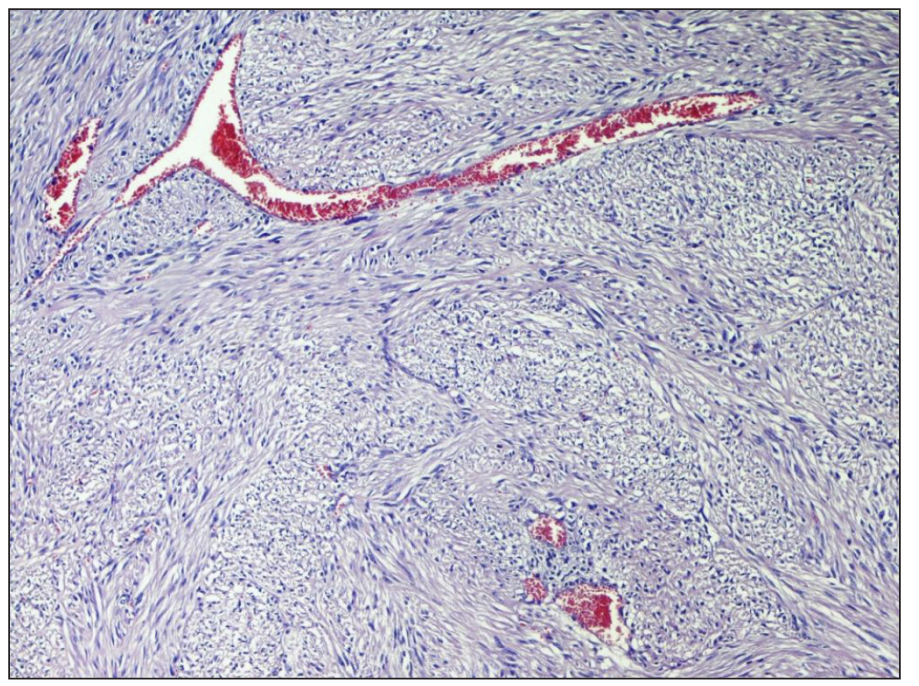

A. Patrón de crecimiento fascicular con orientación en diferentes sentidos constituido por células tumorales fusiformes. Hematoxilina-Eosina.

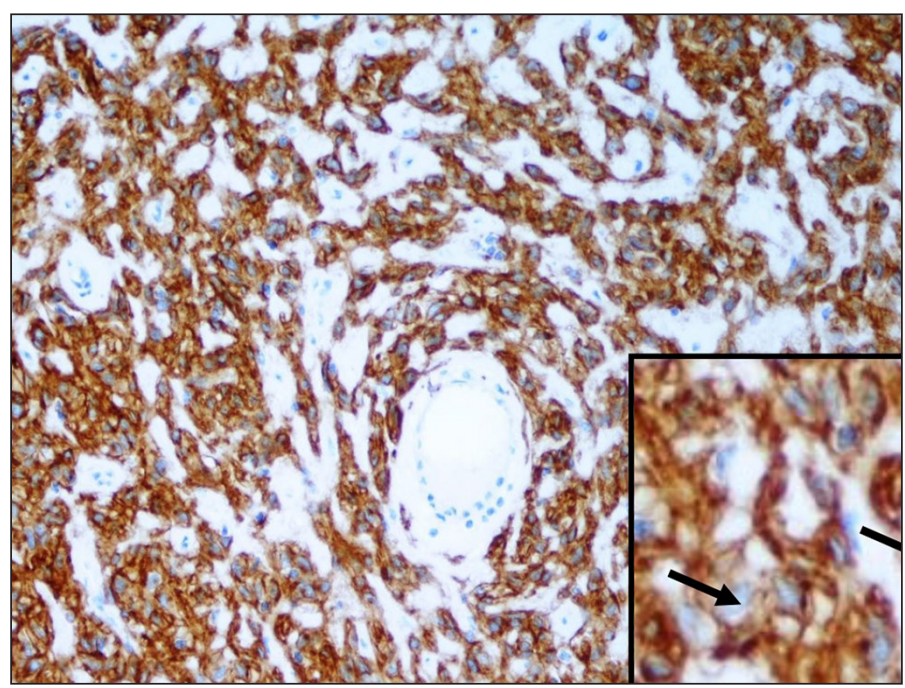

C. Inmunorreactividad citoplasmática intensamente positiva en las células tumorales, núcleo sin marcar (flechas en detalle). Técnica DOG1.



B. Células tumorales fusiformes (flechas) con moderado pleomorfismo nuclear. Hematoxilina-Eosina.

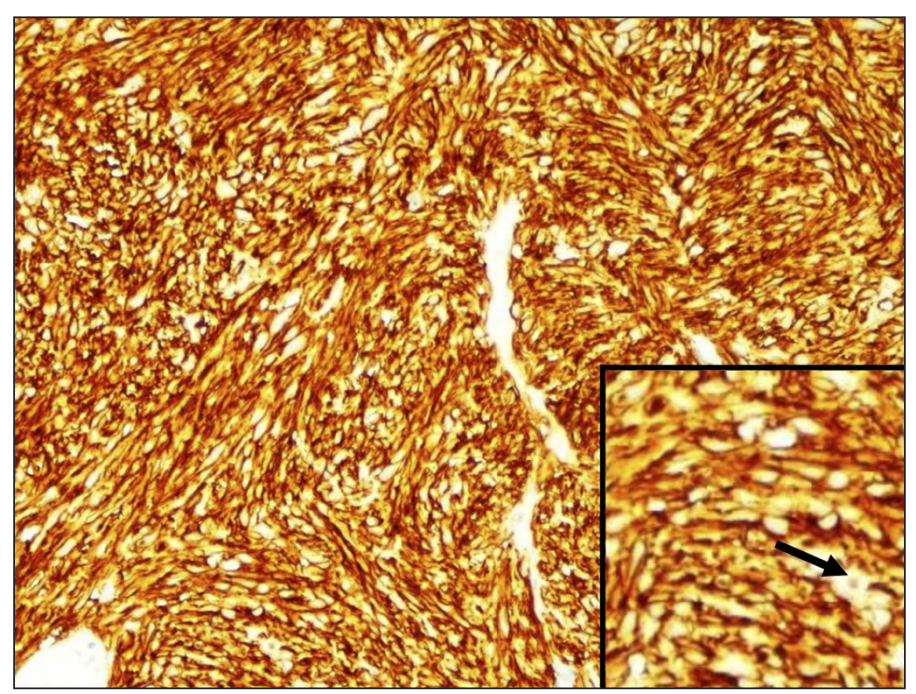

D. Inmunorreactividad intensamente positiva en las células tumorales. Refuerzo de la membrana plasmática, núcleo sin marcar (flechas en detalle) Técnica CD117.

Figura 3. Preparaciones histológicas GIST. A y B) Tinciones con hematoxilina-eosina. C) Inmunorreactividad DOG1. D) Inmunorreactividad CD117.

coincide con los datos observados en la nuestra. Los síntomas más comunes de presentación suelen ser hemorragia, dolor abdominal y obstrucción intestinal, aunque existen GIST que se diagnostican de manera incidental y parecen tener un curso más benigno ${ }^{4}$. En nuestra serie, en cambio, la mayoría se han presentado como hallazgo incidental y efectivamente resultan ser los de menor grado de malignidad. Sólo 1 de los 7 que se presentaron de manera incidental fue metastásico.

Al ser la mayoría de los GIST submucosos, no son accesibles a la toma de biopsias por endoscopia, por lo que el diagnóstico definitivo no se suele establecer hasta después de la cirugía. Además, la punción aspiración con aguja fina (PAAF) no está indicada en principio, ya que aumenta el riesgo de siembra y diseminación tumoral y puede no aportar nada al diagnóstico si se obtiene material hemorrágico o necrótico. Podría estar indicada en los casos en que se plantee neoadyuvancia o en casos de duda diagnóstica en pacientes de alto riesgo ${ }^{5}$.

Existen tres patrones histopatológicos de este tipo de tumores. El más frecuente es el de células fusiformes, seguido del epitelioide y del mixto ${ }^{2}$. Los hallazgos histológicos están íntimamente relacionados con la localización del tumor. Los tumores gástricos son de células fusiformes en la mayoría de los casos. Encontrar un tumor de células epitelioides en intestino delgado se asocia a peor pronóstico ${ }^{6}$.

El diagnóstico definitivo de GIST viene dado por la expresión de c-kit en un tumor gastrointestinal de origen mesenquimal con datos anatomopatológicos característicos de este tipo de tumores. E1 $85 \%$ de los tumores clasificados como 
Tabla 3. Criterios de consenso de pronóstico en los GIST

\begin{tabular}{|lcc|}
\hline & Tamaño (cm) & $\begin{array}{c}\text { Número de mitosis (por 50 } \\
\text { CGA)* }\end{array}$ \\
\hline Muy bajo riesgo & $<2$ & $<5$ \\
Bajo riesgo & $2-5$ & $<5$ \\
Riesgo intermedio & $<5$ & $6-10$ \\
& $5-10$ & $<5$ \\
Alto riesgo & $>5$ & $>5$ \\
& $>10$ & Cualquiera \\
& Cualquiera & $>10$ \\
\hline
\end{tabular}

* CGA: campos de gran aumento.

GIST presentan mutaciones en uno o ambos receptores de tirosin-kinasa, que son el gen KIT o el factor de crecimiento alfa derivado de plaquetas (PDGFR- $\alpha)^{7}$. La tirosin-kinasa es detectada inmunohistoquímicamente por reactividad con el antígeno CD117, que produce una fuerte y difusa tinción citoplasmática. Una pequeña proporción de GIST no reaccionan al CD117 por lo que en algunas ocasiones, es necesario llevar a cabo estudios de mutaciones del receptor, sobre todo si no está claro el diagnóstico o cuando es absolutamente necesario para la instauración del tratamiento ${ }^{8}$. Lo más frecuente es la mutación del exón 11, seguido de la mutación del $9^{6,9}$. En ambos casos podemos encontrar también mutaciones secundarias de los exones 13, 14, 17 ó $18^{7}$.

Recientemente se ha descubierto un nuevo marcador, que resulta ser independiente de las mutaciones KIT o PDGFR- $\alpha$. Se trata de una proteína del canal regulador de calcio y cloro denominada DOG1 cuya presencia parece tener la misma sensibilidad diagnóstica que las mutaciones que se han estado empleando hasta ahora. DOG1 se considera especialmente útil en casos de GIST con tinción CD117 negativa y alta sospecha, o si se piensa en la posibilidad de un falso positivo en la tinción ${ }^{10,11}$. Se ha observado negatividad para ambos marcadores (DOG1 y KIT) en el $2.6 \%$ de los GIST del tracto gastrointestinal ${ }^{10}$.

Es probable que la protein-kinasa $\mathrm{C}$ theta sea expresada en todos los GISTs independientemente de su estado mutacional ${ }^{12,13}$ y pueda utilizarse en un futuro para el diagnóstico de ese 2'6\% de GIST DOG1 y CD117 negativos.

La importancia del diagnóstico preciso radica en la posibilidad de tratamiento satisfactorio en GIST CD117- con Imatinib. El problema estriba en la falta de disponibilidad de los reactivos necesarios en muchos de los hospitales de nuestro entorno.

Los criterios de consenso, establecidos por Fletcher en el 2002, para evaluar el riesgo de los tumores GIST se basan en el tamaño del tumor y en la tasa mitótica ${ }^{2}$ (Tabla 2). Existe una clara correlación entre un tumor de 5 o más $\mathrm{cm}$. y una actividad mitótica aumentada (más de 5 mitosis por 50 CGA) y una mala evolución del paciente con recurrencia local, metástasis y supervivencia reducida ${ }^{8}$. En el mismo año, Miettinen trató de mejorar dicha escala de riesgo añadiendo la localización del tumor ${ }^{14}$, puesto que es un factor de riesgo independiente del tamaño tumoral o el índice mitótico ${ }^{2}$.
Los factores pronósticos de mala evolución del tumor debido a la cirugía son la perforación intraoperatoria y la presencia de enfermedad macroscópica residual ${ }^{15}$. Así, en el 2006 Miettinen y Lasota trataron de incluir estos hallazgos intraoperatorios y la localización del tumor en los criterios de Fletcher, en un intento de mejorar la identificación de aquellos pacientes que pudieran beneficiarse del tratamiento adyuvante ${ }^{6}$.

Del año 2009 a la actualidad se han propuesto nuevos métodos de estadificación para valorar el riesgo de progresión, tales como un nomograma publicado por Gold y cols. ${ }^{16}$, una clasificación TGM por estadios ${ }^{17}$ (donde $\mathrm{T}$ depende del tamaño del tumor, $\mathrm{G}$ del grado de diferenciación y $\mathrm{M}$ de la aparición o no de metástasis), y una clasificación TNM presentada en 2010 por la Unión Internacional Contra el Cáncer (UICC) ${ }^{18}$. Sin embargo, ninguno de estos ha sido aún aceptado y consensuado como lo fue en su momento el sistema de Fletcher.

El tratamiento de elección en la enfermedad primaria es la resección quirúrgica completa (R0: Resección completa sin márgenes afectos, microscópica ni macroscópicamente), ya sea mediante cirugía abierta o cirugía laparoscópica ${ }^{5}$. No son necesarios amplios márgenes de resección, ni linfadenectomías extensas, ya que aunque es muy frecuente la afectación peritoneal y hepática, es improbable la diseminación vía linfática. En ocasiones puede ser necesario realizar resecciones extensas e intervenciones de gran complejidad (amputación abdominoperineal de Miles, Intervención de Whipple) debido, no tanto al tamaño del tumor, como a su localización ${ }^{5,8}$. Este tipo de cirugía tiene una morbimortalidad alta y está asociada a complicaciones graves, como infecciones intraabdominales o dehiscencias anastomóticas $^{8}$. Lo más importante durante la cirugía es evitar la rotura tumoral durante la manipulación, para no provocar implantes tumorales ${ }^{1}$. La rotura del tumor es equivalente a una resección R2 (márgenes macroscópicos afectos) ${ }^{5}$.

Estudios recientes comparan los resultados obtenidos en cirugía abierta frente a los obtenidos en nuevas series de cirugía laparoscópica. La elección de una u otra vía va a depender fundamentalmente de la localización, del tamaño del tumor y de la experiencia del equipo quirúrgico. Se acepta la resección por vía laparoscópica cuando el tamaño no supere los $5 \mathrm{~cm}$, para evitar el riesgo de diseminación peritoneal por manipulación instrumental, y se discute la resección por esta vía en tumores localizados en la unión gastroesófagica, antro-píloro y cara posterior gástrica $^{5,19-21}$.

Los GIST son resistentes a quimioterapia convencional, la radioterapia es completamente inefectiva y, antes de la llegada del Imatinib, se intentó la quimioterapia intraperitoneal o la radioablación (de tumores hepáticos), con resultados poco alentadores.

La quimioembolización arterial hepática, en cambio, ha obtenido buenos resultados para el tratamiento de las metástasis hepáticas secundarias a un GIST. Un estudio de 110 pacientes llevado a cabo en Estados Unidos ha demostrado aumentar tanto la supervivencia libre de recurrencia hepática como la supervivencia global en pacientes sometidos a este procedimiento ${ }^{22}$.

En diversas series se ha demostrado que el $20-50 \%$ de los pacientes con tumores inicialmente resecables presentan recidiva o metástasis. Las metástasis pueden aparecer hasta pasados 15 años después de la primera intervención quirúrgica, por lo que es necesario realizar un seguimiento a largo plazo $^{6}$. 
En la época pre-Imatinib (antes del 2001), la supervivencia tras cirugía supuestamente curativa a los 5 años oscilaba entre el $32-78 \%$, disminuyendo a un $19-63 \%$ a los 10 años ${ }^{8}$.

En cambio, desde la introducción del Imatinib en el postoperatorio de estos pacientes, los resultados son muy diferentes. Se obtienen índices de supervivencia libre de recurrencia de enfermedad significativamente superiores en los grupos sometidos a tratamiento adyuvante ${ }^{23,24}$

El imatinib (Gleevec $\AA$; Novartis Pharma AG, Basel, Switzerland) es un principio activo, aprobado en 2002 en España, con actividad antineoplásica no relacionado ni química ni farmacológicamente con otros fármacos antineoplásicos, por lo que representa una nueva entidad química y farmacológica en el tratamiento del cáncer ${ }^{25}$. Sus indicaciones autorizadas, en pacientes adultos, son:

a) El tratamiento de la leucemia mieloide crónica (LMC) cromosoma Filadelfia positivo (bcr-abl) en fase crónica tras fallo de tratamiento con interferon alfa, en fase acelerada o crisis blástica.

b) El tratamiento de la leucemia linfoblástica aguda cromosoma Filadelfia positivo (LLA $\mathrm{Ph}+)$ de diagnóstico reciente (integrado con quimioterapia) o en casos refractarios o recaídas.

c) El tratamiento de síndromes mielodisplásicos/mieloproliferativos (SMD/SMP) asociados con el reordenamiento del gen del receptor del factor de crecimiento derivado de las plaquetas (PDGFR).

d) El tratamiento del síndrome hipereosinofílico (SHE) avanzado y/o leucemia eosinofílica crónica (LEC) con reordenación de FIP1L1-PDGFR.

e) El tratamiento de tumores GIST malignos no resecables y/o metastásicos CD 117 positivos

f) El tratamiento de tumores GIST operados y clasificados de intermedio o alto riesgo, como terapia adyuvante para aumentar la supervivencia libre de recurrencia ${ }^{23,24,26}$.

Imatinib es un inhibidor de la proteina tirosina-kinasa que bloquea la función de las proteínas anormales presentes en la LMC, inhibiendo de forma selectiva la proliferación e induciendo la apoptosis de las líneas celulares ber-abl positivas, así como en células leucémicas nuevas de LMC cromosoma Filadelfia positivo y en pacientes con leucemia linfoblástica aguda. También se ha visto que inhibe el receptor tirosinkinasa para el factor de crecimiento derivado de plaquetas (PDGFR- $\alpha$ ) y del factor de células troncales (FCT) y C-kit. No obstante, la utilidad de dicho fármaco varía en función de la mutación presente en el tumor. Los mejores resultados se han obtenido en aquellos que presentan mutaciones del exón 11, siendo considerablemente mayor el riesgo relativo de progresión de la enfermedad en los GIST con cualquier otra mutación ${ }^{7}$.

Imatinib es un fármaco bien tolerado en general y la mayor parte de los efectos secundarios son de intensidad leve a moderada y comprenden: edema, náuseas, diarrea, calambres, fatiga, erupción cutánea, dispepsia, granulocitopenia, elevación de la GOT o GPT. Los efectos secundarios más graves e infrecuentes incluyen hemorragias intestinales y peritoneales, probablemente debidas a necrosis y hemorragias intratumorales, leucopenia, hipotiroidismo e insuficiencia cardíaca congestiva ${ }^{11}$.

En los últimos años se han llevado a cabo numerosos estudios de los cuales referimos los más significativos para establecer la indicación de Imatinib adyuvante (Tabla 4) $)^{23,24}$.

Se ha discutido durante tiempo cuál es la dosis óptima de Imatinib adyuvante y se han llevado a cabo numerosos ensayos tras los cuales se ha establecido que la dosis recomendada es de $400 \mathrm{mg}$ /día en tumores que presentan mutaciones del exón $11^{15}$ y de $800 \mathrm{mg} /$ día en aquellos con mutaciones del exón $9^{11}$. Se debe continuar el tratamiento sólo si se observa una respuesta al tratamiento inicial dentro de las 12 primeras semanas. La Tomografía por Emisión de positrones (PET) y la Tomografía Computerizada (TC) son las pruebas recomendadas para su valoración, siendo necesario realizarlas previamente en situación basal ${ }^{19}$. La PET permite observar la existencia de respuesta en cuestión de semanas (incluso desde el octavo día de tratamiento ${ }^{27,28}$, sin embargo en la TC es necesario mayor tiempo de exposición al fármaco. Si se observa en la TC un cambio en la densidad del tumor (medido en unidades Hounsfiled) es indicativo de buena respuesta ${ }^{15}$. No obstante, se han obtenido resultados de algunos estudios en los que la PET ha infraestimado la respuesta al tratamiento en algunos pacientes, en con-

Tabla 4. Estudios de adyuvancia en GIST ${ }^{23,24}$.

Estudio Fase III. ACOSOG Z9001 American College of Surgeons Oncology Group and the National Cancer Institute (USA).

Adyuvancia con Imatinib en GIST de bajo, intermedio y alto riesgo, tras resección completa.

Método: Estudio randomizado, doble ciego, multicéntrico. Tratamiento con Imatinib durante 1 año.

Pacientes: 713.

Resultados: Aumento de la supervivencia al año de un $83 \%$ a un $98 \%$ y en dos años de un $71 \%$ a un $90 \%$.

Estudio fase III. (China)

Compara supervivencia libre de recurrencia en el primer, segundo y tercer año en pacientes tratados con Imatinib frente a placebo.

Método: Tratamiento durante 3 años, comenzando a las 4-12 semanas de la cirugía.

Pacientes: 105.

Resultados: Aumento de la supervivencia libre de recurrencia al año de un $90 \%$ a un $100 \%$, en dos años de un $57 \%$ a un $96 \%$ y a los 3 años de un $48 \%$ a un $89 \%$.

Estudio fase III del grupo escandinavo SSG XVIII/AIO

Compara supervivencia libre de recurrencia en dos grupos tratados con Imatinib durante distinto periodo de tiempo.

Método: Tratamiento adyuvante con Imatinib en dosis de $400 \mathrm{mg} /$ día, durante 12 meses en un grupo y 36 meses en el otro.

Pacientes: 397.

Resultado: Aumento de la supervivencia libre de recurrencia significativamente superior en el grupo que recibe tratamiento durante 36 meses,

comparado con el grupo que lo recibe durante 12 meses $(\mathrm{HR}=0,45[0,22,0,89], \mathrm{p}=0,0187)$. 
Tabla 5. Estudios de neoadyuvancia en GIST.

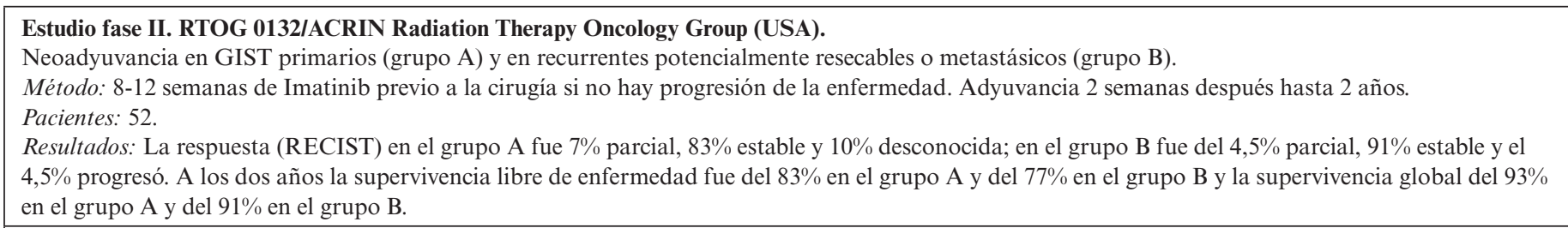

\section{Estudio fase III. BFR 14}

Pacientes con GIST localmente avanzados o metastásicos que no habían progresado al cabo de 1 año de tratamiento con Imatinib se aleatorizaron a la interrupción o continuación del tratamiento.

Se hizo un subanálisis de forma retrospectiva incluyendo sólo a los 25 pacientes con enfermedad localmente avanzada que no habían sido intervenidos previamente.

Método: 1 año de tratamiento con Imatinib, si no hay progresión de la enfermedad. Cirugía posterior en 9 casos, antes de cumplir el año.

Pacientes: 25.

Resultados: Aumento de la supervivencia libre de recurrencia en los pacientes sometidos a cirugía posterior a la neoadyuvancia. $67 \%$ a los 3 años.

traposición con la TC y la anatomía patológica de la pieza de la posterior resección ${ }^{29}$.

No se recomienda un aumento de la dosis de Imatinib en aquellos pacientes que desarrollan enfermedad progresiva tras una respuesta inicial.

La duración óptima del tratamiento está siendo estudiada en numerosos ensayos clínicos (Tabla 4) ${ }^{23,24}$, aunque algunos ya concluyen que un año no es suficiente ${ }^{23}$.

Diversos estudios demostraron que más del $80 \%$ de los pacientes con GIST inoperables o metastásicos tratados con Imatinib y seguidos durante al menos 9 meses, han presentado respuesta favorable al tratamiento con disminución del tamaño del tumor del $50 \%$ o más o ausencia de progresión de la enfermedad $^{8,30}$

Considerando estos buenos resultados en pacientes con tumores GISTs malignos no resecables y/o metastásicos, se está planteando la posibilidad del empleo de Imatinib como tratamiento neoadyuvante en pacientes que son, o podrían ser, candidatos a cirugía. Existen diferentes estudios que se están llevando a cabo en la actualidad sobre el tratamiento neoadyuvante (Tabla 5) $)^{31,32}$.

La neoadyuvancia disminuye el tamaño tumoral y el edema. $\mathrm{Su}$ utilidad radica en la disminución del riesgo de rotura y de

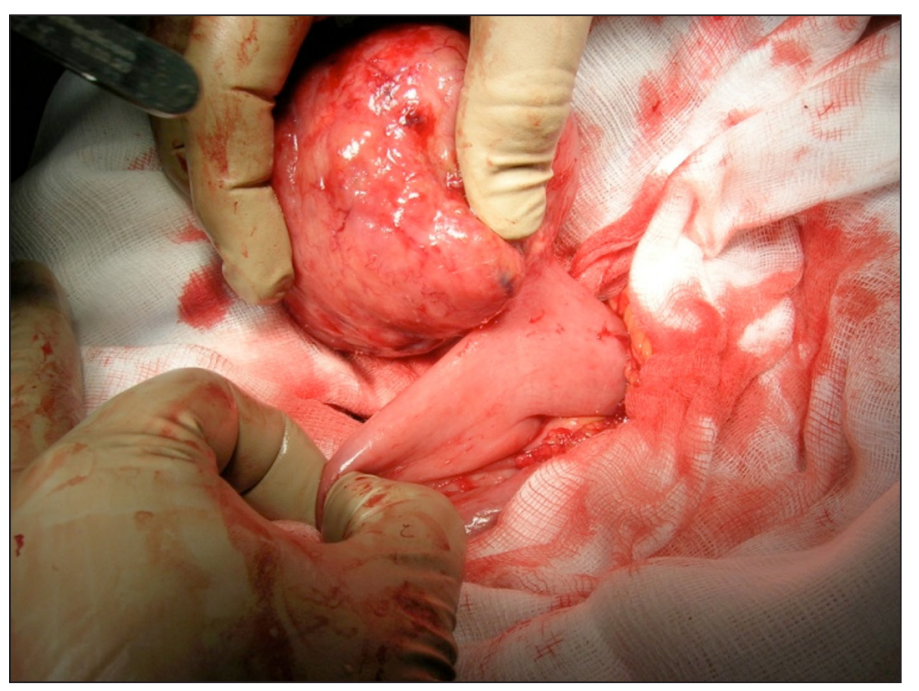

Figura 4. GIST situado en el borde antimesentérico del primer asa yeyunal. sangrado durante la cirugía ${ }^{5}$, puesto que son dos factores de riesgo conocidos de recurrencia de la enfermedad.

Se considera que un paciente presenta resistencia al tratamiento con Imatinib si no hay respuesta tras 3-6 meses de terapia. Existe un mayor índice de resistencia primaria en el caso de los GIST con mutaciones del exón 9 y del gen PDGFR- $\alpha^{33}$. En estos casos de resistencia está aprobado el empleo de Sunitinib malate (Sutent ${ }^{\circledR}$, Pfizer, New York, USA) como terapia de segunda línea ${ }^{33,34}$.

Sunitinib es el que mejores resultados ha mostrado después del Imatinib, pero están en estudio otros como el everolimus, HSP90 y Flavopiridol. Los estudios de fase I/II están obteniendo mejores resultados con el uso conjunto de everolimus (actúa inhibiendo mTOR) y un inhibidor de tirosin-kinasa, sobre todo al emplearlo como terapia de tercera línea ${ }^{35}$.

Actualmente también se investiga el uso de sorafenib, dasatinib, motesanib y nilotinib en GIST resistentes al tratamiento con imatinib y sunitinib ${ }^{36}$.

\section{CONCLUSIONES}

El descubrimiento de nuevos métodos inmunohistoquímicos nos ha permitido diferenciar a los GIST del resto de tumores mesenquimales del tracto gastrointestinal.

La importancia no ha sido meramente a efectos de clasificación, ya que la aparición del Imatinib que actúa selectivamente sobre el receptor c-kit, ha convertido a los GIST en el primer modelo de tumor sólido que puede tratarse de una manera eficaz.

En el intestino delgado todos los tumores salvo uno han sido de intermedio o alto grado y, aún teniendo presente que el número de casos analizados es escaso, parece ir en línea con la probable mayor malignidad de los tumores localizados en el intestino delgado frente a los gástricos.

La incidencia estimada es de 1 nuevo caso por año para una población de 100.000 habitantes.

Hasta que los resultados de los estudios en curso sobre la terapia neoadyuvante de imatinib estén disponibles, se pueden sugerir las siguientes consideraciones desde nuestro punto de vista:

a) En tumores GIST operables, se debería realizar primero cirugía y considerar al paciente para tratamiento 
con Imatinib como terapia adyuvante en casos de intermedio o alto grado, de resección incompleta, rotura del tumor de forma intraoperatoria o factores de alto riesgo.

b) En GIST marginalmente resecables o en casos de GIST recurrente operable o metastático, considerar el tratamiento con Imatinib previo a la cirugía.

Actualmente parece que la dosis óptima de Imatinib para los tumores con mutaciones en el exón 11 es de $400 \mathrm{mg} /$ día y de $800 \mathrm{mg}$ /día en los tumores con mutaciones del exón 9 . Se están llevando a cabo estudios para determinar la duración óptima, los efectos secundarios a corto y largo plazo y el empleo en terapia neoadyuvante.

Debido a la baja incidencia de este tipo de neoplasias son necesarios estudios multicéntricos para poder inferir resultados estadísticamente significativos.

\section{BIBLIOGRAFIA}

1. DeMatteo RP, Lewis JJ, Leung D, Mudan SS, Woodruff JM, Brennan MF. Two hundred gastrointestinal stromal tumors: recurrence patterns and prognostic factors for survival. Ann Surg 2000; 231:51-8.

2. Fletcher CDM, Berman JJ, Corless C, et al. Diagnosis of gastrointestinal stromal tumors: a consensus approach. Int J Surg Pathol. 2002 Apr;10(2):81-9.

3. Blay JY. A decade of tyrosine kinase inhibitor therapy: Historical and current perspectives on targeted therapy for GIST. Cancer Treat Rev. 2011 Aug;37(5):373-84.

4. Hassan I, You YN, Shyyan R, Dozois EJ, Smyrk TC, Okuno SH, Schleck CD, Hodge DO, Donohue JH. Surgically Managed Gastrointestinal Stromal Tumors: A Comparative and Prognostic Analysis. Ann Surg Oncol. 2008 Jan;15(1):52-9.

5. Fernández JA, Sánchez-Cánovas ME, Parrilla P. Controversias en el tratamiento quirúrgico de los tumores del estroma gastrointestinal (GIST) primarios. Cir Esp. 2010 Aug;88(2):69-80. Epub 2010 May 24.

6. Miettinen M, Lasota J. Gastrointestinal Stromal Tumors: Review on Morphology, Molecular Pathology, Prognosis, and Differential Diagnosis. Arch Pathol Lab Med. 2006 Oct;130(10):1466-78.

7. Blay JY. New paradigms in gastrointestinal stromal tumour management. Ann Oncol. 2009 May;20 Suppl 1:i18-24.

8. Eisenberg BL, Judson I. Surgery and Imatinib in the Management of GIST: Emerging Approaches to Adjuvant and Neoadjuvant Therapy. Ann Surg Oncol 2004;11:465-475.

9. Díaz Delgado M, Hernández Amate A, Pereira Gallardo S, Jaramillo S, Virizuela Echaburu JA, González-Cámpora RJ. Gastrointestinal Stromal Tumors: Morphological, Immunohistochemical and Molecular Changes Associated with Kinase Inhibitor Therapy. Pathol Oncol Res. 2011 Sep;17(3):455-61.

10. Miettinen M, Wang ZF, Lasota J. DOG1 antibody in the differential diagnosis of gastrointestinal stromal tumors: a study of 1840 cases. Am J Surg Pathol. 2009 Sep;33(9):1401-8.

11. Demetri GD, Benjamin RS, Blanke CD, Blay JY, Casali P, Choi H, Corless CL, Debiec-Rychter M, DeMatteo RP, Ettinger DS, Fisher GA, Fletcher CD, Gronchi A, Hohenberger P, Hughes M, Joensuu H, Judson I, Le Cesne A, Maki RG, Morse M, Pappo AS, Pisters PW, Raut CP, Reichardt P, Tyler DS, Van den Abbeele AD, von Mehren M, Wayne JD, Zalcberg J; NCCN Task Force. NCCN Task Force Report: Update on the Management of Patients with GIST. J Natl Compr Canc Netw. 2007 Jul;5 Suppl 2:S1-29; quiz S30.

12. Duensing A, Joseph NE, Medeiros F, et al. Protein kinase C theta (PKCtheta) expression and constitutive activation in gastrointestinal stromal tumors (GISTs). Cancer Res. 2004;64:5127-5131.
13. Blay P, Astudillo A, Buesa JM, et al. Protein kinase C theta is highly expressed in gastrointestinal stromal tumors but not in other mesenchymal neoplasias. Clin Cancer Res. 2004;10:4089-4095.

14. Miettinen M, El-Rifai W, H L Sobin L, Lasota J. Evaluation of Malignancy and Prognosis of Gastrointestinal Stromal Tumors: A Review. Hum Pathol 2002 May;33(5):478-83.

15. Casali PG, Blay JY; ESMO/CONTICANET/EUROBONET Consensus Panel of Experts. Gastrointestinal stromal tumours: ESMO Clinical recommendatios for diagnosis, treatment and folow-up. Ann Oncol. 2010 May;21 Suppl 5:v98-102.

16. Gold JS, Gönen M, Gutiérrez A, Broto JM, García-del-Muro X, Smyrk TC, Maki RG, Singer S, Brennan MF, Antonescu CR, Donohue JH, DeMatteo RP. Development and validation of a prognostic nomogram for recurrencefree survival after complete surgical resection of localized, primary gastrointestinal stromal tumor (GIST)- A retrospective analysis. Lancet Oncol. 2009 Nov;10(11):1045-52.

17. Woodall CE 3rd, Brock GN, Fan J, Byam JA, Scoggins CR, McMasters KM, Martin RC 2nd. An evaluation of 2537 gastrointestinal stromal tumors for a proposed clinical staging system. Arch Surg. 2009;144(7):670-678.

18. International union against cancer (UICC). TNM classification of malignant tumours. $7^{\text {th }}$ ed. Sobin LH, Wittekind Ch., eds. New York: Wiley; 2010.

19. Poveda A, Artigas V, Casado A, Cervera J, García Del Muro X, Antonio López-Guerrero J, López-Pousa A, Maurel J, Ortega L, Ramos R, Romero I, Safont MJ, Martín J; Grupo Español de Investigación en Sarcomas (GEIS). Guía práctica clínica en los tumores estromales gastrointestinales (GIST)actualización 2008. Cir Esp. 2008 Oct;84 Suppl 1:1-21.

20. Raut CP, Ashley SW. How I do it: surgical management of gastrointestinal stromal tumors. J Gastrointest Surg. 2008 Sep;12(9):1592-9.

21. Alam I, Kheradmand F, Alam S, Jamil A, Wilson I, Hurley M. Laparoscopic Management of Acutely Presenting Gastrointestinal Stromal Tumors: A Study of 9 Cases and Review of Literature. J Laparoendosc Adv Surg Tech A. 2007 Oct;17(5):626-33.

22. Kobayashi K, Gupta S, Trent JC, Vauthey JN, Krishnamurthy S, Ensor J, Ahrar K, Wallace MJ, Madoff DC, Murthy R, McRae SE, Hicks ME. Hepatic Artery Chemoembolization for 110 Gastrointestinal Stromal Tumors. Cancer. 2006 Dec 15;107(12):2833-41.

23. Li J, Gong JF, Wu AW, Shen L. Postoperative imatinib in patients with intermediate or high risk gastrointestinal stromal tumor. Eur J Surg Oncol. 2011 Apr;37(4):319-24.

24. Dematteo RP, Ballman KV, Antonescu CR, Maki RG, Pisters PW, Demetri GD, Blackstein ME, Blanke CD, von Mehren M, Brennan MF, Patel S, McCarter MD, Polikoff JA, Tan BR, Owzar K; American College of Surgeons Oncology Group (ACOSOG) Intergroup Adjuvant GIST Study Team. Placebo controlled randomized trial of adjuvant imatinib mesylate following the resection, localized, primary GIST. Lancet. 2009 Mar 28;373(9669):1097-104.

25. Glivec $($. Ficha Técnica de la especialidad. The European Agency for the Evaluation of Medicinal Products (EMEA). Committee for Propietary Medicinal Products (CPMP). European Public Assesment Report (EPAR) Glivec ${ }^{\circledR}$.

26. Eisenberg BL, Smith KD. Adjuvant and neoadjuvant therapy for primary GIST. Cancer Chemother Pharmacol. 2011 Jan;67 Suppl 1:S3-8.

27. Alberini JL, Al Nakib M, Wartski M, Gontier E, Cvitkovic F, Rixe O, Rougier P, Pecking AP. The role of PET scan in gastrointestinal stromal tumors. Gastroenterol Clin Biol. 2007 Jun-Jul;31(6-7):585-93.

28. Van den Abbeele AD, Gatsonis C, de Vries DJ, Melenevsky Y, Szot-Barnes A, Yap JT, Godwin AK, Rink L, Huang M, Blevins M, Sicks J, Eisenberg B, Siegel BA. ACRIN 6665/RTOG 0132 phase II trial of neoadjuvant imatinib mesylate for operable malignant gastrointestinal stromal tumor: monitoring with 18F-FDG PET and correlation with genotype and GLUT4 expression. J Nucl Med. 2012 Apr;53(4):567-74. Epub 2012 Mar 1.

29. Andtbacka RH, Ng CS, Scaife CL, Cormier JN, Hunt KK, Pisters PW, Pollock RE, Benjamin RS, Burgess MA, Chen LL, Trent J, Patel SR, Raymond K, Feig BW. Surgical Resection of Gastrointestinal Stromal Tumors After Treatment with Imatinib. Ann Surg Oncol. 2007 Jan;14(1):14-24.

30. Demetri GD, von Mehren M, Blanke CD, et al. Efficacy and safety of imatinib mesylate in advanced gastrointestinal stromal tumors. N Engl J Med 2002;347:472-80.

31. Eisenberg BL, Harris J, Blanke CD, Demetri GD, Heinrich MC, Watson JC, Hoffman JP, Okuno S, Kane JM, von Mehren M. Phase II trial of neoadjuvant- 


\section{Tumores del estroma gastrointestinal (GIST): serie del Hospital Central de la Defensa Gómez Ulla}

adjuvant imatinib mesylate (IM) for advanced primary and metastatic: recurrent operable GIST: early results of RTOG 0132. J Surg Oncol. 2009 Jan 1;99(1):42-7.

32. Blesius A, Cassier PA, Bertucci F, Fayette J, Ray-Coquard I, Bui B, Adenis A, Rios M, Cupissol D, Pérol D, Blay JY, Le Cesne A. Neoadjuvant imatinib in patients with locally advanced non metastatic GIST in the prospective BFR14 trial. BMC Cancer. 2011 Feb 15;11:72.

33. Duensing S, Duensing A. Targeted therapies of gastrointestinal stromal tumors (GIST): The next frontiers. Biochem Pharmacol. 2010 Sep 1;80(5):575-83.

34. Demetri GD, van Oosterom AT, Garrett CR, Blackstein ME, Shah MH, Verweij J, McArthur G, Judson IR, Heinrich MC, Morgan JA, Desai J, Fletcher CD, George S, Bello CL, Huang X, Baum CM, Casali PG. Efficacy and safety of sunitinib in patients with advanced gastrointestinal stromal tumour after failure of imatinib: a randomised controlled trial. Lancet. 2006 Oct 14;368(9544):1329-38.

35. Schöffski P, Reichardt P, Blay JY, Dumez H, Morgan JA, Ray-Coquard I, Hollaender N, Jappe A, Demetri GD. A phase I-II study of everolimus (RAD001) in combination with imatinib in patients with imatinib-resistant gastrointestinal stromal tumors. Ann Oncol. 2010 Oct;21(10):1990-8. Epub 2010 May 27.

36. Lamba G, Gupta R, Lee B, Ambrale S, Liu D. Current management and prognostic features for gastrointestinal stromal tumor (GIST). Exp Hematol Oncol. 2012 Jun 18;1(1):14. 